\title{
The medical information age
}

$\mathrm{H}_{\mathrm{n}}^{\mathrm{a}}$ ave you ever had trouble getting a history of past illnesses and wondered if this might lead to unwanted surprises in treatment or in the operating room? Here is the answer.

Patients carry computer discs of their medical records. When they need medical care the entire record is there, immediately. Gone are the days of, "I don't know what drugs I'm on, call my doctor." Now it is all there on the screen with immediate access. As physicians, we would know what tests had been done, and the results. This should be a cost saving as it would result in fewer tests being repeated when patients are transferred or attend different physicians and hospitals. It would all be on the disc carried by the patient.

Why should the patient carry the disc? Easy access and control. Should the doctor have a copy? Yes, if the patient agrees, and having this information on computer would eliminate one of the most expensive aspects of practice overhead: access and storage of paper files.

A danger to confidentiality and too easy to copy the discs? This problem is eliminated because patients have their own disc, and can decide who to give it to, and what should be restricted. The disc can be encoded to limit access, all or in part. Can a patient handle the truth, or is it better the patient not know? If a patient does not want to know, he doesn't have to look, or even have a disc.

Forget what the doctor said on your last visit? Look it up on your computer.

Concerned that psychiatric care would be compromised? Don't put it on the computer. Leave this idea for physical illness only.

It's possible, easy and beneficial, so why don't we have this already? Here are a few possibilities:

1. Patients might think when they have a second opinion it would be better if the physician did not know their past history but approach the problem independently.

2. There is good reason to think present health is what counts, not past health.

3. Information about past health has consequences, especially with insurance companies and in compensation questions.

4. It would discriminate against people who do not use computers.

5. It would create a divide - those with and without a disc of their medical records.
6. It would lead to

two sets of

records. Gone

would be the

term 'difficult

patient'. Of

course, these

comments no

longer appear in

charts now

anyway.

7. Rarely, medical

records are

reconstructed. This

is a very bad idea

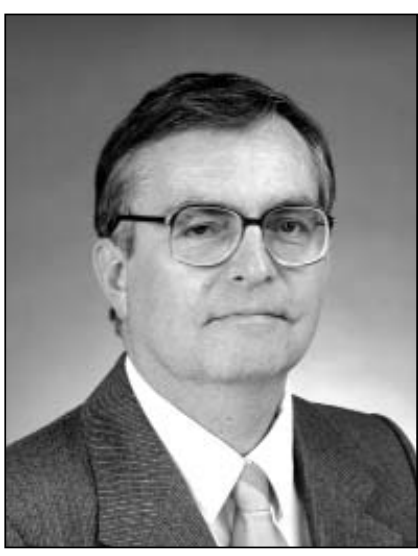

John R Taylor and diagnosing reconstruction is a well-developed

forensic science. How hard would it be to diagnose a

reconstructed computer health record?

We have an easy way to keep track of past health history, a way that should save a lot of time and benefit patients by adding access to information which might be crucial in health care. What if we added a program that red flags potential drug interactions, a list entered by the patient of all the patent medicines they are taking, a built-in differential diagnosis and the history becomes even more practical. Can't type? No problem. Just have a list of possibilities and chose from the list.

What about the medical records department? They would have less transcription to do, but would be just as busy doing other things; converting paper documents to disc, updating discs with present hospital records and producing the health record discs patients carry. Microfilming would be a thing of the past.

Worried about losing your heath care disc? Have it subcutaneously implanted; a great help if you are brought unconscious into the emergency room and might save your life some day. All we need is something to read it through the skin.

Don't look for any of this to happen any time soon. There are too many problems with confidentiality, supposed or real, and the health care system is occupied with other, more urgent, situations right now. It is still a good idea, so why don't we start by encouraging patients to carry their own paper records on health care visits, and as physicians and surgeons why don't we keep a copy of our own heath records for our own future visits to the doctor? Or, maybe this is just a hypochondriac's dream. 